EUROPEAN JOURNAL OF PURE AND APPLIED MATHEMATICS

Vol. 12, No. 2, 2019, 332-347

ISSN 1307-5543 - www.ejpam.com

Published by New York Business Global

\title{
On weak graded rings II
}

\author{
Najla Al-Subaie ${ }^{1}$, M. M. Al-Shomrani ${ }^{2, *}$ \\ ${ }^{1}$ Department of Mathematics, Taif University, Taif, Saudi Arabia \\ ${ }^{2}$ King Abdulaziz University, Faculty of Science, P.O.Box 80203, Jeddah 21589, Saudi Arabia
}

\begin{abstract}
The $\mathfrak{G}$-weak graded rings are rings graded by a set $\mathfrak{G}$ of left coset representatives for the left action of a subgroup $H$ of a finite group $X$. The main aim of this article is to study the concept of $\mathfrak{G}$-weak graded rings and continue the investigation of their properties. Moreover, some results concerning $\mathfrak{G}$-weak graded rings of fractions are derived. Finally, some additional examples of $\mathfrak{G}$-weak graded rings are provided.
\end{abstract}

2010 Mathematics Subject Classifications: 16W50, 13A02, 16D25

Key Words and Phrases: Weak graded rings, fully weak graded rings, left coset representatives, graded rings of fractions, homogeneous elements.

\section{Introduction}

Recall that, for a group $X$ and a ring $R, R$ is called $X$-graded if, for each element $g$ in the group $X$, there is an additive subgroup $R_{g}$ of $R$, such that $R=\bigoplus_{g \in X} R_{g}$ and, for all $g, h \in X$, we have $R_{g} R_{h} \subseteq R_{g h}$.

Group graded rings as well as Clifford theory for group graded rings were studied and their properties were investigated by many mathematicians, see for exampl [8], [9],[10], [12], [16], [21] and [22]. Nevertheless, rings and modules can be graded by using semigroups instead of groups leading to more general results as we can see in [1], [11], [13], [14], [15] and [19].

Many ways have been used to investigate the properties of these rings. In [7], Cohen and Montgomery introduced an interesting way using duality theorems, see also [5]. Another useful way is the associated graded ring construction which states that for a valuation ring $R$, we can associate a ring $R_{G}$ graded by the valuation group $G$. This ring seems to be easier to be studied and the properties can be lifted back from $R_{G}$ to $R$. This way is one of the motivations for studying graded rings, see [16] for more details. Moreover, some mathematicians introduced categorical methods to study these graded rings such as the study of separable functors introduced in [17] and [20]. Most of these methods have been

* Corresponding author.

DOI: https://doi.org/10.29020/nybg.ejpam.v12i2.3380

Email addresses: njlalsubaie@gmail.com (N. Al-Subaie), malshomrani@hotmail.com (M. Al-Shomrani) 
introduced for the finite group-grading. However, more additional investigations have been done considering the infinite case, see for example [2].

In [6], a fixed set $\mathfrak{G}$ of left coset representatives for the left action of a subgroup $H$ on a group $X$ was constructed and a binary operation on $\mathfrak{G}$, which has a left identity and the right division property, was defined. This binary operation is not associative in a travail way. However, the associativity was considered by using a "cocycle" $f: \mathfrak{G} \times \mathfrak{G} \longrightarrow H$. The dependence on the choice of representatives was shown as follows: For a given subgroup $H$ of a group $X$, different sets of representatives $\mathfrak{G}$ and $\underline{\mathfrak{G}}$ for the left cosets can be chosen. These cosets are related by an arbitrary function $\gamma: X / H \longrightarrow H$, so that if $s \in \mathfrak{G}$ then $\gamma([s]) s \in \underline{\mathfrak{G}}$, where $[s]$ denotes the coset $H s$.

In [3], the concept of group graded rings was extended by using a set $\mathfrak{G}$ of left coset representatives with specific binary operation. This new concept was called $\mathfrak{G}$-weak graded rings. In [4], some properties of weak graded rings were investigated. Moreover, graded rings by using the product $H \times \mathfrak{G}$ were also discussed.

In this article, we consider the $\mathfrak{G}$-weak graded rings and continue the investigation of their properties. More specifically, for a finite group $X$, a subgroup $H$, a fixed set of left coset representatives $(\mathfrak{G}, *)$ and a $\mathfrak{G}$-weak graded ring $R$ with unity, the following results are proved: (i) If $K$ is a subring of $R$ containing all of its $\mathfrak{G}$-homogeneous elements, then $K$ is a $\mathfrak{G}$-weak graded subring. (ii) If $x$ is a unit element such that $x \in R_{s}$ for some $s \in \mathfrak{G}$, then $x^{-1} \in R_{s^{L}}$ where $s^{L}$ is the left inverse of $s$. (iii) $W G r U(R)$, the set of all weak graded units of $R$, is a subgroup of $U(R)$. Moreover, some results considering the $\mathfrak{G}$-weak graded rings of fraction are proved. Finally, some additional examples of $\mathfrak{G}$-weak graded rings are introduced.

Throughout this article, we shall assume that all groups are finite, all rings are commutative with unities and all vector spaces are finite dimensional.

\section{Preliminaries}

In this section, we list some important definitions and results that will be used later in this article.

Definition 1. [6] Let $X$ be a group and $H$ be a subgroup of $X$. We call $\mathfrak{G} \subset X$ a set of left coset representatives if, for every $x \in X$, there is a unique $s \in \mathfrak{G}$ such that $x \in H s$. In addition, we call the decomposition $x=u s$, for $u \in H$ and $s \in \mathfrak{G}$, the unique factorization of $x$.

In what follows, $\mathfrak{G}$ stands for a fixed set of left coset representatives for the action of the subgroup $H$ of $X$ on the group $X$ and $e$ is the identity element in $X$.

Definition 2. [6] For elements $s, t \in \mathfrak{G}$ we define $f(s, t) \in H$ and $s * t \in \mathfrak{G}$ by the unique factorization st $=f(s, t)(s * t)$ in $X$, where $f$ is the cocycle map. Moreover, the functions $\triangleright: \mathfrak{G} \times H \rightarrow H$ and $\triangleleft: \mathfrak{G} \times H \rightarrow \mathfrak{G}$ are defined by the unique factorization $s u=(s \triangleright u)(s \triangleleft u)$ for $s, s \triangleleft u \in \mathfrak{G}$ and $u, s \triangleright u \in H$. 
The binary operation $*$ on $\mathfrak{G}$ has a unique left identity $e_{\mathfrak{G}} \in \mathfrak{G}$ and satisfying the right division property, i.e., for all $s, t \in \mathfrak{G}$ there is a unique solution $p \in \mathfrak{G}$ satisfying the equation $p * s=t[6]$. If $e \in \mathfrak{G}$, then $e_{\mathfrak{G}}=e$ is also a right identity. Also, there is a unique left inverse $s^{L}$ for every $s \in \mathfrak{G}$ satisfying the equation $s^{L} * s=e_{\mathfrak{G}}$.

Proposition 1. [6] The following identities between $(\mathfrak{G}, *)$ and $f$ are satisfied for all $s, t, p \in \mathfrak{G}$ and all $u, v \in H$ :

$$
\begin{gathered}
s \triangleright(t \triangleright u)=f(s, t)((s * t) \triangleright u) f(s \triangleleft(t \triangleright u), t \triangleleft u)^{-1}, \\
(s * t) \triangleleft u=(s \triangleleft(t \triangleright u)) *(t \triangleleft u), \\
s \triangleright u v=(s \triangleright u)((s \triangleleft u) \triangleright v), \\
s \triangleleft u v=(s \triangleleft u) \triangleleft v, \\
f(p, s) f(p * s, t)=(p \triangleright f(s, t)) f(p \triangleleft f(s, t), s * t)
\end{gathered}
$$

and

$$
(p \triangleleft f(s, t)) *(s * t)=(p * s) * t .
$$

Proposition 2. [6] The following identities between $(\mathfrak{G}, *)$ and $f$ are satisfied for all $t \in \mathfrak{G}$ and all $v \in H$ :

$$
\begin{gathered}
e_{\mathfrak{G}} \triangleleft v=e_{\mathfrak{G}}, \quad e_{\mathfrak{G}} \triangleright v=e_{\mathfrak{G}} v e_{\mathfrak{G}}^{-1}, \quad t \triangleright e=e, \quad t \triangleleft e=t, \\
f\left(e_{\mathfrak{G}}, t\right)=e_{\mathfrak{G}}, \quad t \triangleright e_{\mathfrak{G}}^{-1}=f\left(t \triangleleft e_{\mathfrak{G}}^{-1}, e_{\mathfrak{G}}\right)^{-1} \quad \text { and } \quad\left(t \triangleleft e_{\mathfrak{G}}^{-1}\right) * e_{\mathfrak{G}}=t .
\end{gathered}
$$

Now, we include the definition of $\mathfrak{G}$-weak graded rings and some related results form [3] where the binary operation $*$ is as in Definition 2 .

Definition 3. [3], [4] Let $X$ be a group, $H$ be a subgroup of $X$ and $(\mathfrak{G}, *)$ be a fixed set of left coset representatives for the left action of $H$ on $X . A$ ring $R$ is called a $\mathfrak{G}$-weak graded ring if

$$
R=\bigoplus_{s \in \mathfrak{G}} R_{s}
$$

and

$$
R_{s} R_{t} \subseteq R_{s * t} \quad \text { for all } s, t \in \mathfrak{G},
$$

where $R_{s}$ is an additive subgroup for each $s \in \mathfrak{G}$. If (2) is replaced by

$$
R_{s} R_{t}=R_{s * t} \quad \text { for all } s, t \in \mathfrak{G},
$$

then, $R$ is called a fully (or strongly) $\mathfrak{G}$-weak graded ring. 
It can be noted that any ring $R$ can be put into a $\mathfrak{G}$-weak graded ring by placing $R=R_{e_{\mathfrak{G}}}$ and $R_{s}=0$ for all $s \in \mathfrak{G}$ with $s \neq e_{\mathfrak{G}}$. This is called the trivial $\mathfrak{G}$-weak graded ring.

Proposition 3. [3] Let $\mathfrak{G}$ be a fixed set of left coset representatives for a subgroup $H$ of a group $X$ and $R$ be a $\mathfrak{G}$-weak graded ring with identity. Then, $1_{R} \in R_{e_{\mathfrak{G}}}$, where $1_{R}$ is the multiplicative identity of $R$.

Proposition 4. [3] Let $R$ be a $\mathfrak{G}$-weak graded ring. Then the $e_{\mathfrak{G}}$-component $R_{\mathfrak{E}_{\mathfrak{G}}}$ is a subring of $R$.

\section{3. $\mathfrak{G}$-weak graded subrings and related results}

In [3] and [4], the definition of weak graded rings was given and some of their properties were derived. In this section, we continue the investigation of the properties of weak graded rings. We start by giving a definition for a $\mathfrak{G}$-homogeneous element of a $\mathfrak{G}$-weak graded ring $R$.

Definition 4. Let $R$ be a $\mathfrak{G}$-weak graded ring. Then, a non-zero element $r_{s} \in R$ is said to be a weak graded or $\mathfrak{G}$-homogeneous element of grade $s$ if there exists an $s$-component $R_{s}$ of $R$ such that $r_{s} \in R_{s}$. The grade of $r_{s}$ is denoted by $\left\langle r_{s}\right\rangle=s$. The set of all $\mathfrak{G}$-homogeneous elements of $R$ is defined and written as $h(R)=\cup_{s \in \mathfrak{G}} R_{s}$.

Remark 1. By Definition 3, every element $r \in R$ has a unique decomposition written as $r=\sum_{s \in \mathfrak{G}} r_{s}$ with $r_{s} \in R_{s}$ for all $s \in \mathfrak{G}$. These $\left\{r_{s}\right\}_{s \in \mathfrak{G}}$ are called the $\mathfrak{G}$-homogeneous components of $r$. However, the sum $\sum_{s \in \mathfrak{G}} r_{s}$ is finite (in other words almost all $r_{s}$ are zero).

Definition 5. Let $K$ be a subring of a $\mathfrak{G}$-weak graded ring $R$. Then, $K$ is said to be a $\mathfrak{G}$-weak graded subring of $R$ if $K$ itself is a $\mathfrak{G}$-weak graded ring.

Theorem 1. Let $K$ be a subring of a $\mathfrak{G}$-weak graded ring $R$. If $K$ contains all the $\mathfrak{G}$ homogeneous components for each $k \in K$, then $K$ is a $\mathfrak{G}$-weak graded subring of $R$.

Proof. Clearly, we can write $K$ as $K=\sum_{s \in \mathfrak{G}} K_{s}$ as additive subgroups. Then for every $k \in K$, we have $k=\sum_{s \in \mathfrak{G}} k_{s}$ where $k_{s} \in K_{s}$ for all $s \in \mathfrak{G}$. We need to prove that $K=\bigoplus_{s \in \mathfrak{G}} K_{s}$ and $K_{s} K_{t} \subseteq K_{s * t}$ which we do as follows:

(i) $K=\bigoplus_{s \in \mathfrak{G}} K_{s}$.

Since $K$ is a subring of $R$ and $R=\bigoplus_{s \in \mathfrak{G}} R_{s}$, we have $K_{s}=K \cap R_{s}$ for all $s \in$ $\mathfrak{G}$. Also, as $R$ is a $\mathfrak{G}$-weak graded ring, the direct sum condition implies that $R_{s} \cap\left(\sum_{t \in \mathfrak{G}} R_{t}\right)=\{0\}$ for all $s \in \mathfrak{G}$ with $s \neq t$, which consequently implies that $K_{s} \cap\left(\sum_{t \in \mathfrak{G}} K_{t}\right)=\{0\}$. Thus, $K=\bigoplus_{s \in \mathfrak{G}} K_{s}$.

(ii) $K_{s} K_{t} \subseteq K_{s * t}$.

Let $K_{s}=K \cap R_{s}$ and $K_{t}=K \cap R_{t}$ for some $s$ and $t$ in $\mathfrak{G}$. Hence,

$$
K_{s} K_{t}=\left(K \cap R_{s}\right)\left(K \cap R_{t}\right) \subseteq K \cap\left(R_{s} R_{t}\right) \subseteq K \cap\left(R_{s * t}\right)=K_{s * t},
$$


which completes the proof.

It can be noted that the last two lines in part $(i)$ of the proof can be, equivalently, written as follows:

$$
K_{s} \cap\left(\sum_{t \in \mathfrak{G}} K_{t}\right)=\left(K \cap R_{s}\right) \cap\left(\sum_{t \in \mathfrak{G}} K \cap R_{t}\right)=K \cap\left(R_{s} \cap \sum_{t \in \mathfrak{G}} R_{t}\right)=K \cap\{0\}=\{0\},
$$

for all $s \in \mathfrak{G}$ with $s \neq t$.

Corollary 1. A subring $K$ of a $\mathfrak{G}$-weak graded ring $R$ is a $\mathfrak{G}$-weak graded subring if and only if $K=\bigoplus_{s \in \mathfrak{G}}\left(K \cap R_{s}\right)$.

Theorem 2. Let $X$ be a group, $H$ be a subgroup of $X$ and $(\mathfrak{G}, *)$ be a fixed set of left coset representatives. Suppose that $\mathfrak{G}$ has a right inverse $s^{R}$ for each $s \in \mathfrak{G}$ and that $s^{R}=s^{L}=s^{-1}$. Then, the $\mathfrak{G}$-weak graded ring $R$ is fully if and only if $1_{R} \in R_{s^{L}} R_{s}$ for all $s \in \mathfrak{G}$.

Proof. Let $R$ be a fully $\mathfrak{G}$-weak graded ring. Then, we have

$$
1_{R} \in R_{e_{\mathfrak{G}}}=R_{s^{L} * s}=R_{s^{L}} R_{s} .
$$

On the other hand, let $1_{R} \in R_{s} L R_{s}$. Since $R$ is $\mathfrak{G}$-weak graded ring, we have $R_{t} R_{s} \subseteq R_{t * s}$. So, we only need to show that $R_{t * s} \subseteq R_{t} R_{s}$ which we do as follows:

$$
\begin{aligned}
& R_{t * s}=R_{t * s} 1_{R} \subseteq R_{t * s} R_{s^{L}} R_{s} \subseteq R_{(t * s) * s^{L}} R_{s}=R_{\left(t \triangleleft f\left(s, s^{L}\right)\right) *\left(s * s^{L}\right)} R_{s} \\
& =R_{\left(t \triangleleft f\left(s, s^{L}\right)\right) * e_{\mathfrak{G}}} R_{s} \\
& =R_{\left(t \triangleleft e_{\mathfrak{G}}^{-1}\right) * e_{\mathfrak{G}}} R_{s} \\
& =R_{t} R_{s} \text {, }
\end{aligned}
$$

as required.

Theorem 3. If $R$ is a $\mathfrak{G}$-weak graded ring and $I$ is an ideal of $R_{e_{\mathfrak{G}}}$, then $I R \cap R_{e_{\mathfrak{G}}}=I$.

Proof. We have to show that $I \subseteq I R \cap R_{e_{\mathfrak{G}}}$ and $I R \cap R_{e_{\mathfrak{G}}} \subseteq I$. It is obvious that $I \subseteq I R \cap R_{e_{\mathfrak{G}}}$ as, for any $i \in I$, we can write $i=i 1_{R} \in I R$ as well as $i \in R_{e_{\mathfrak{G}}}$ since $I \subseteq R_{e_{\mathfrak{G}}}$.

So, it is enough to show that $I R \cap R_{e_{\mathfrak{G}}} \subseteq I$ which we do as follows: Suppose that $x \in I R \cap R_{e_{\mathfrak{G}}}$ which implies that $x \in I R$ and $x \in R_{e_{\mathfrak{G}}}$. Since $x \in I R$, we can write $x$ as

$$
x=\sum_{\text {finite }} i r,
$$

for $i \in I$ and $r \in R$. As $R$ is a $\mathfrak{G}$-weak graded ring, equation (4) can be rewritten as

$$
x=\sum_{\text {finite }} i r_{s}, \quad \text { for } \quad s \in \mathfrak{G},
$$

where $r_{s} \in R_{s}$. Also, as $x \in R_{e_{\mathfrak{G}}}$, hence $x$ can be written as $x=i r_{e_{\mathfrak{G}}}$. Thus, $x \in I$ since $I$ is an ideal of $R_{e_{\mathfrak{G}}}$. Therefore, $I R \cap R_{e_{\mathfrak{G}}} \subseteq I$ which completes the proof. 
Definition 6. For a $\mathfrak{G}$-weak graded ring $R$, a unit $x \in U(R)$ is said to be weak graded unit or a $\mathfrak{G}$-homogeneous unit if $x \in R_{s}$ for some $s \in \mathfrak{G}$, where $U(R)$ is the group of all units in $R$. The set of all weak graded units in $R$ is denoted by $W G r U(R)$.

Theorem 4. Let $R$ be a $\mathfrak{G}$-weak graded ring with unity and let $x$ be an element in $U(R)$. If $x \in R_{s}$, for some $s \in \mathfrak{G}$, then $x^{-1} \in R_{s^{L}}$.

Proof. Let $x \in R_{s}$ for some $s \in \mathfrak{G}$ and let $x^{-1}=\sum_{t \in \mathfrak{G}} r_{t}$ where $r_{t} \in R_{t}$ such that all but a finite number of them are zero. Hence,

$$
r_{t} x \in R_{t} R_{s} \subseteq R_{t * s} \quad \text { for any } t \in \mathfrak{G} .
$$

Thus, $x^{-1} x=\sum_{t \in \mathfrak{G}} r_{t} x$ is the unique expansion for $x^{-1} x$ in the $\operatorname{direct} \operatorname{sum} R=\bigoplus_{t \in \mathfrak{G}} R_{t * s}$ which is equivalent to $R=\bigoplus_{s \in \mathfrak{G}} R_{s}$ since $\mathfrak{G}$ is closed under the binary operation *. But, on the other side, we have

$$
x^{-1} x=1_{R} \in R_{e_{\mathfrak{G}}}=R_{s^{L} * s} .
$$

Consequently, $\sum_{t \in \mathfrak{G}} r_{t} x=1_{R}$ which implies that $r_{t}=0$ for all $t \neq s^{L}$. So, if we put $t=s^{L}$ we get $r_{s^{L}} x=1_{R}$. Therefore, $r_{s^{L}}=x^{-1} \in R_{s^{L}}$ as required.

Theorem 5. If $R$ is a $\mathfrak{G}$-weak graded ring, then the set of all its weak graded units, $W G r U(R)$, is a subgroup of $U(R)$ and the map $\langle-\rangle: W G r U(R) \longrightarrow \mathfrak{G}$, satisfies the homomorphism property of groups with $\langle-\rangle^{-1}\left(e_{\mathfrak{G}}\right)=W G r U\left(R_{e_{\mathfrak{G}}}\right)$, where $\langle-\rangle$ is the $\mathfrak{G}_{-}$ grade.

Proof. First, to show that the set $\operatorname{WGr} U(R)$ is a subgroup of $U(R)$, let $x, y \in$ $W G r U(R)$. Then $x \in R_{s}$ and $y \in R_{t}$ for some $s, t \in \mathfrak{G}$. Now, since $R$ is a $\mathfrak{G}$-weak graded ring, we have

$$
x y \in R_{s} R_{t} \subseteq R_{s * t} .
$$

Also, as $\mathfrak{G}$ is closed under the operation $*$, we can put $s * t=p$ for some $p \in \mathfrak{G}$. Hence, $x y$ is weak graded for all $x, y \in W G r U(R)$. In addition, as $x, y \in W G r U(R) \subseteq U(R)$ and that $U(R)$ is a group under the ring multiplication, then $x y \in U(R)$. Thus $x y$ is a weak graded unit for any $x, y \in W G r U(R)$, i.e., $x y \in W G r U(R)$. Moreover, if $x \in W G r U(R)$, then, by Theorem $4, x^{-1} \in W G r U(R)$.

Next, to show that the map $\langle-\rangle$ satisfies the homomorphism property of groups, let $x, y \in W G r U(R)$ and suppose that $x \in R_{s}$ and $y \in R_{t}$ for some $s, t \in \mathfrak{G}$, i.e. $\langle x\rangle=s$ and $\langle y\rangle=t$. Thus, $x y \in R_{s} R_{t} \subseteq R_{s * t}=R_{p}$, for some $p \in \mathfrak{G}$. Consequently,

$$
\langle x y\rangle=p=s * t=\langle x\rangle *\langle y\rangle .
$$


Finally,

$$
\begin{array}{rlrl}
\langle-\rangle^{-1}\left(e_{\mathfrak{G}}\right) & =\{x \in W \operatorname{Wr} U(R): & \left.\langle x\rangle=e_{\mathfrak{G}}\right\} \\
& =\{x \in W \operatorname{Wr} U(R): & & \left.x \in R_{e_{\mathfrak{G}}}\right\} \\
& =W \operatorname{WrU}\left(R_{e_{\mathfrak{G}}}\right), & &
\end{array}
$$

as required.

It should be noted that $\mathfrak{G}$ is, in general, neither a group nor even a monoid.

Proposition 5. If a ring $R$ is a $\mathfrak{G}$-weak graded ring, then the conjugation in $R$ defines an action given by

$$
\varphi_{R}: r_{e_{\mathfrak{G}}}, x \longrightarrow r_{e_{\mathfrak{G}}}^{x}=x^{-1} r_{e_{\mathfrak{G}}} x
$$

of the group $\operatorname{WGr} U(R)$ as automorphisms of the subring $R_{e_{\mathfrak{G}}}$, where $r_{e_{\mathfrak{G}}} \in R_{e_{\mathfrak{G}}}$ and $x \in W G r U(R)$.

Proof. The conjugation by a weak graded unit $x$ in $R_{s}$ for some $s \in \mathfrak{G}$ is an automorphism of the ring $R$ satisfying:

$$
R_{e_{\mathfrak{G}}}^{x}=x^{-1} R_{e_{\mathfrak{G}}} x \subseteq R_{s^{L}} R_{e_{\mathfrak{G}}} R_{s} \subseteq R_{s^{L}} R_{e_{\mathfrak{G}} * s}=R_{s} R_{s} \subseteq R_{e_{\mathfrak{G}}} .
$$

Since $\operatorname{WGr} U(R)$ is a group, the proof is completed.

\section{4. $\mathfrak{G}$-weak graded rings of fractions}

We start this section by recalling the following well known results [18]:

(a) Let $R$ be a ring and $K$ be a multiplicatively closed subset of $R$ such that $1_{R} \in K, 0 \notin K$. Then the left ring of fractions with respect to $K, K^{-1} R$, exists if and only if $R$ satisfies the left Ore conditions with respect to $K$, i.e.:

(i) If $r k=0$, for some $k \in K$ and $r \in R$, then there is an element $k^{\prime} \in K$ such that $k^{\prime} r=0$.

(ii) For $r \in R$ and $k \in K$, there are elements $r^{\prime} \in R$ and $k^{\prime} \in K$ such that $k^{\prime} r=r^{\prime} k$.

If Ore conditions with respect to $K$ are satisfied, then

$$
K^{-1} R=R_{K}=\left\{\frac{r}{k}: r \in R, k \in K\right\} .
$$

The addition and multiplication operations on $R_{K}$ are defined, respectively, by $\frac{r}{k}+\frac{r^{\prime}}{k^{\prime}}=$ $\frac{k^{\prime} r+k r^{\prime}}{k k^{\prime}}$ and $\frac{r}{k} \cdot \frac{r^{\prime}}{k^{\prime}}=\frac{r_{1} r^{\prime}}{k_{1}^{\prime} k}$ for $k_{1}^{\prime} \in K, r_{1} \in R$ with $k_{1}^{\prime} r=r_{1} k^{\prime}$.

(b) For every $M$ in $R$-Mod, we can construct a fraction $K^{-1} M$ which is a left $K^{-1} R$ module. Moreover, $K^{-1} M \cong K^{-1} R \otimes_{R} M$.

Now, we prove the following lemma which will be used to prove the the next theorem. 
Lemma 1. Let $X$ be a group, $H$ be a subgroup of $X$ and $\mathfrak{G} \subset X$ be a set of left coset representatives. Then, for all $s, t \in \mathfrak{G}$, the multiplication $s * t$ in $\mathfrak{G}$ has a left inverse given by:

$$
(s * t)^{L}=\left(t^{L} \triangleleft f\left(s^{L} \triangleleft f(s, t), s * t\right)^{-1}\right) *\left(s^{L} \triangleleft f(s, t)\right) .
$$

Proof. We have to show that

$$
\left(\left(t^{L} \triangleleft f\left(s^{L} \triangleleft f(s, t), s * t\right)^{-1}\right) *\left(s^{L} \triangleleft f(s, t)\right)\right) *(s * t)=e_{\mathfrak{G} .}
$$

To do so, we start with the left hand side of equation (5) as follows:

$$
\begin{aligned}
& \left(\left(t^{L} \triangleleft f\left(s^{L} \triangleleft f(s, t), s * t\right)^{-1}\right) *\left(s^{L} \triangleleft f(s, t)\right)\right) *(s * t)= \\
& \left(\left(t^{L} \triangleleft f\left(s^{L} \triangleleft f(s, t), s * t\right)^{-1}\right) \triangleleft f\left(s^{L} \triangleleft f(s, t), s * t\right)\right) *\left(\left(s^{L} \triangleleft f(s, t)\right) *(s * t)\right)= \\
& \left(t^{L} \triangleleft f\left(s^{L} \triangleleft f(s, t), s * t\right)^{-1} f\left(s^{L} \triangleleft f(s, t), s * t\right)\right) *\left(\left(s^{L} * s\right) * t\right)= \\
& \quad t^{L} * t=e_{\mathfrak{G} .}
\end{aligned}
$$

The right division property yields $\left(t^{L} \triangleleft f\left(s^{L} \triangleleft f(s, t), s * t\right)^{-1}\right) *\left(s^{L} \triangleleft f(s, t)\right)=(s * t)^{L}$ as required.

Theorem 6. Let $R$ be a $\mathfrak{G}$-weak graded ring and $K$ be a multiplicatively closed set of $\mathfrak{G}$-homogeneous elements not containing 0 . Then the localization $R_{K}$ can be written as the direct sum of its $\mathfrak{G}$-components as follows:

$$
R_{K}=\bigoplus_{s \in \mathfrak{G}}\left(R_{K}\right)_{s}, \quad \text { (as additive subgroups) }
$$

with,

$$
R_{K}=\left\{\frac{r}{k}: r \in R, k \in K\right\}
$$

and

$$
\left(R_{K}\right)_{s}=\left\{\frac{r}{k} \in R_{K}: r \text { and } k \text { are } \mathfrak{G} \text {-homogeneous and }\langle k\rangle^{L} *\langle r\rangle=s\right\}
$$

such that $f(s, t)=f(s, p)$ for all $s, t, p \in \mathfrak{G}$, where $\langle k\rangle,\langle r\rangle$ are the $\mathfrak{G}$-grades of $k$ and $r$ respectively.

Proof. We begin the proof by showing that $\left(R_{K}\right)_{s}$ is an additive subgroup of $R_{K}$ as follows: If $\frac{r}{k}, \frac{r^{\prime}}{k^{\prime}} \in\left(R_{K}\right)_{s}$, then $\langle k\rangle^{L} *\langle r\rangle=s=\left\langle k^{\prime}\right\rangle^{L} *\left\langle r^{\prime}\right\rangle$. Hence, $\frac{r}{k}+\frac{r^{\prime}}{k^{\prime}}=\frac{k^{\prime} r+k r^{\prime}}{k k^{\prime}}$. 
Consequently,

$$
\begin{aligned}
\left\langle\frac{k^{\prime} r+k r^{\prime}}{k k^{\prime}}\right\rangle & =\left\langle k k^{\prime}\right\rangle^{L} *\left\langle k r^{\prime}\right\rangle \\
& =\left(\langle k\rangle *\left\langle k^{\prime}\right\rangle\right)^{L} *\left(\langle k\rangle *\left\langle r^{\prime}\right\rangle\right) \\
& =\left(\left(\left\langle k^{\prime}\right\rangle^{L} \triangleleft f\left(\langle k\rangle^{L} \triangleleft f\left(\langle k\rangle,\left\langle k^{\prime}\right\rangle\right),\langle k\rangle *\left\langle k^{\prime}\right\rangle\right)^{-1}\right) *\left(\langle k\rangle^{L} \triangleleft f\left(\langle k\rangle,\left\langle k^{\prime}\right\rangle\right)\right)\right) *\left(\langle k\rangle *\left\langle r^{\prime}\right\rangle\right) \\
& =\left(\left(\left\langle k^{\prime}\right\rangle^{L} \triangleleft f\left(\langle k\rangle^{L} \triangleleft f\left(\langle k\rangle,\left\langle k^{\prime}\right\rangle\right),\langle k\rangle *\left\langle k^{\prime}\right\rangle\right)^{-1}\right) \triangleleft f\left(\langle k\rangle^{L} \triangleleft f\left(\langle k\rangle,\left\langle k^{\prime}\right\rangle\right),\langle k\rangle *\left\langle r^{\prime}\right\rangle\right)\right) \\
& *\left(\langle k\rangle^{L} \triangleleft f\left(\langle k\rangle,\left\langle k^{\prime}\right\rangle\right) *\left(\langle k\rangle *\left\langle r^{\prime}\right\rangle\right)\right) \\
& =\left(\left\langle k^{\prime}\right\rangle^{L} \triangleleft f\left(\langle k\rangle^{L} \triangleleft f\left(\langle k\rangle,\left\langle k^{\prime}\right\rangle\right),\langle k\rangle *\left\langle k^{\prime}\right\rangle\right)^{-1} f\left(\langle k\rangle^{L} \triangleleft f\left(\langle k\rangle,\left\langle k^{\prime}\right\rangle\right),\langle k\rangle *\left\langle r^{\prime}\right\rangle\right)\right. \\
& \left.*\left(\left(\langle k\rangle^{L} *\langle k\rangle\right) *\left\langle r^{\prime}\right\rangle\right)\right) \\
& =\left\langle k^{\prime}\right\rangle^{L} *\left(e_{\mathfrak{G}} *\left\langle r^{\prime}\right\rangle\right)=\left\langle k^{\prime}\right\rangle^{L} *\left\langle r^{\prime}\right\rangle=s .
\end{aligned}
$$

Also, if $\frac{r}{k} \in\left(R_{K}\right)_{s}$, then $-\left(\frac{r}{k}\right)=\frac{-r}{k} \in\left(R_{K}\right)_{s}$. Indeed, if $\frac{r}{k} \in\left(R_{K}\right)_{s}$, then $\langle k\rangle^{L} *\langle r\rangle=s=$ $\langle k\rangle^{L} *\langle-r\rangle$ as $r$ is a $\mathfrak{G}$-homogeneous element, i.e. $r$ is contained in fixed component and since each component are additive subgroup of $R$ yields $-r$ has the same $\mathfrak{G}$-grade. Hence, $\left(R_{K}\right)_{s}$ is an additive subgroup of $R_{K}$ for all $s \in \mathfrak{G}$.

Next, we have to show that $R_{K}=\bigoplus_{s \in \mathfrak{G}}\left(R_{K}\right)_{s}$. It is obvious that $R_{K}=\sum_{s \in \mathfrak{G}}\left(R_{K}\right)_{s}$. So, let $\frac{r}{k} \in\left(R_{K}\right)_{s_{j}} \bigcap\left\{\left(R_{K}\right)_{s_{1}}+\ldots+\left(R_{K}\right)_{s_{j-1}}+\left(R_{K}\right)_{s_{j+1}}+\ldots+\left(R_{K}\right)_{s_{n}}\right\}$ for all $s_{j} \neq$ $s_{1}, \ldots, s_{j-1}, s_{j+1}, \ldots, s_{n}$. Thus,

$$
\langle k\rangle^{L} *\langle r\rangle=s_{j} \quad \text { and } \quad\langle k\rangle^{L} *\langle r\rangle=s_{i},
$$

where $i=1, \ldots, j-1, j+1, \ldots, n$. This means that, either $\left(R_{K}\right)_{s_{j}}=\left(R_{K}\right)_{s_{i}}$ which is a contradiction or $\frac{r}{k}=0_{R_{K}}$ which implies $R_{K}=\bigoplus_{s \in \mathfrak{G}}\left(R_{K}\right)_{s}$ as required.

\section{Additional examples of $\mathfrak{G}$-weak graded rings}

In this section, we give additional examples of $\mathfrak{G}$-weak and fully $\mathfrak{G}$-weak graded rings that are not trivially constructed.

Example 1. Consider a ring $R$ to be the ring of all $2 \times 2$ matrices over the field $\mathbb{R}$, i.e.,

$$
R=M_{2}(\mathbb{R})=\left\{\left(\begin{array}{ll}
a & b \\
c & d
\end{array}\right) \quad: \quad a, b, c, d \in \mathbb{R}\right\} .
$$

Let $X$ be the dihedral group $D_{6}=\left\langle x, y: x^{6}=y^{2}=1, x y=y x^{5}>\right.$ and $H$ be the non-normal subgroup $\left\{1, x^{3}, y, x^{3} y\right\}$. Choose $\mathfrak{G}=\left\{1, x, x^{5}\right\}$ to be the set of left coset representatives. Then the $*$ and $f$ operations as well as the actions $\triangleleft$ and $\triangleright$ are given by the following tables:

Thus, $R=R_{1} \oplus R_{x} \oplus R_{x^{5}}$, where

$$
R_{1}=\left\{\left(\begin{array}{ll}
a & 0 \\
0 & d
\end{array}\right): a, d \in \mathbb{R}\right\}
$$


Table 1: $*$ and $f$ operations.

\begin{tabular}{|l||l|l|l|}
\hline$*$ & 1 & $\mathrm{x}$ & $x^{5}$ \\
\hline \hline 1 & 1 & $\mathrm{x}$ & $x^{5}$ \\
$\mathrm{x}$ & $\mathrm{x}$ & $x^{5}$ & 1 \\
$x^{5}$ & $x^{5}$ & 1 & $\mathrm{x}$ \\
\hline
\end{tabular}

\begin{tabular}{|l||l|l|l|}
\hline$f$ & 1 & $\mathrm{x}$ & $x^{5}$ \\
\hline \hline 1 & 1 & 1 & 1 \\
$\mathrm{x}$ & 1 & $x^{3}$ & 1 \\
$x^{5}$ & 1 & 1 & $x^{3}$ \\
\hline
\end{tabular}

Table 2: $\triangleright$ and $\triangleleft$ actions.

\begin{tabular}{|l||l|l|l|l|}
\hline$s \triangleright u$ & 1 & $x^{3}$ & $\mathrm{y}$ & $x^{3} y$ \\
\hline \hline 1 & 1 & $x^{3}$ & $\mathrm{y}$ & $x^{3} y$ \\
$\mathrm{x}$ & 1 & $x^{3}$ & $\mathrm{y}$ & $x^{3} y$ \\
$x^{5}$ & 1 & $x^{3}$ & $\mathrm{y}$ & $x^{3} y$ \\
\hline
\end{tabular}

\begin{tabular}{|l||l|l|l|l|}
\hline$s \triangleleft u$ & 1 & $x^{3}$ & $\mathrm{y}$ & $x^{3} y$ \\
\hline \hline 1 & 1 & 1 & 1 & 1 \\
$\mathrm{x}$ & $\mathrm{x}$ & $\mathrm{x}$ & $x^{5}$ & $x^{5}$ \\
$x^{5}$ & $x^{5}$ & $x^{5}$ & $x$ & $x$ \\
\hline
\end{tabular}

$$
R_{x}=\left\{\left(\begin{array}{ll}
0 & 0 \\
c & 0
\end{array}\right) \quad: \quad c \in \mathbb{R}\right\}
$$

and

$$
R_{x^{5}}=\left\{\left(\begin{array}{ll}
0 & b \\
0 & 0
\end{array}\right) \quad: \quad b \in \mathbb{R}\right\}
$$

Moreover, the inclusion property $R_{s} R_{t} \subseteq R_{s * t}$ is satisfied for all $s, t \in \mathfrak{G}$. This can be detailed as follows:

(i) $R_{1} R_{1} \subseteq R_{1 * 1}=R_{1}$, as for all $\left(\begin{array}{cc}a_{1} & 0 \\ 0 & d_{1}\end{array}\right)$, $\left(\begin{array}{cc}a_{2} & 0 \\ 0 & d_{2}\end{array}\right) \in R_{1}$, we have

$$
\left(\begin{array}{cc}
a_{1} & 0 \\
0 & d_{1}
\end{array}\right)\left(\begin{array}{cc}
a_{2} & 0 \\
0 & d_{2}
\end{array}\right)=\left(\begin{array}{cc}
a_{1} a_{2} & 0 \\
0 & d_{1} d_{2}
\end{array}\right) \in R_{1}=R_{1 * 1}
$$

(ii) $R_{1} R_{x} \subseteq R_{1 * x}=R_{x}$, as for all $\left(\begin{array}{ll}a & 0 \\ 0 & d\end{array}\right) \in R_{1}$ and $\left(\begin{array}{ll}0 & 0 \\ c & 0\end{array}\right) \in R_{x}$, we have

$$
\left(\begin{array}{ll}
a & 0 \\
0 & d
\end{array}\right)\left(\begin{array}{ll}
0 & 0 \\
c & 0
\end{array}\right)=\left(\begin{array}{cc}
0 & 0 \\
d c & 0
\end{array}\right) \in R_{x}=R_{1 * x}
$$

(iii) $R_{1} R_{x^{5}} \subseteq R_{1 * x^{5}}=R_{x^{5}}$, as for all $\left(\begin{array}{ll}a & 0 \\ 0 & d\end{array}\right) \in R_{1}$ and $\left(\begin{array}{ll}0 & b \\ 0 & 0\end{array}\right) \in R_{x^{5}}$, we have

$$
\left(\begin{array}{ll}
a & 0 \\
0 & d
\end{array}\right)\left(\begin{array}{ll}
0 & b \\
0 & 0
\end{array}\right)=\left(\begin{array}{cc}
0 & a b \\
0 & 0
\end{array}\right) \in R_{x^{5}}=R_{1 * x^{5}}
$$

(iv) $R_{x} R_{1} \subseteq R_{x * 1}=R_{x}$, as for all $\left(\begin{array}{ll}0 & 0 \\ c & 0\end{array}\right) \in R_{x}$ and $\left(\begin{array}{ll}a & 0 \\ 0 & d\end{array}\right) \in R_{1}$, we have

$$
\left(\begin{array}{ll}
0 & 0 \\
c & 0
\end{array}\right)\left(\begin{array}{ll}
a & 0 \\
0 & d
\end{array}\right)=\left(\begin{array}{cc}
0 & 0 \\
c a & 0
\end{array}\right) \in R_{x}=R_{x * 1} .
$$


(v) $R_{x} R_{x} \subseteq R_{x * x}=R_{x^{5}}$, as for all $\left(\begin{array}{cc}0 & 0 \\ c_{1} & 0\end{array}\right),\left(\begin{array}{cc}0 & 0 \\ c_{2} & 0\end{array}\right) \in R_{x}$, we have

$$
\left(\begin{array}{cc}
0 & 0 \\
c_{1} & 0
\end{array}\right)\left(\begin{array}{cc}
0 & 0 \\
c_{2} & 0
\end{array}\right)=\left(\begin{array}{cc}
0 & 0 \\
0 & 0
\end{array}\right) \in R_{x^{5}}=R_{x * x}
$$

(vi) $R_{x} R_{x^{5}} \subseteq R_{x * x^{5}}=R_{1}$, as for all $\left(\begin{array}{cc}0 & 0 \\ c & 0\end{array}\right) \in R_{x}$ and $\left(\begin{array}{cc}0 & b \\ 0 & 0\end{array}\right) \in R_{x^{5}}$, we have

$$
\left(\begin{array}{ll}
0 & 0 \\
c & 0
\end{array}\right)\left(\begin{array}{cc}
0 & b \\
0 & 0
\end{array}\right)=\left(\begin{array}{cc}
0 & 0 \\
0 & c b
\end{array}\right) \in R_{1}=R_{x * x^{5}} .
$$

(vii) $R_{x^{5}} R_{1} \subseteq R_{x^{5} * 1}=R_{x^{5}}$, as for all $\left(\begin{array}{cc}0 & b \\ 0 & 0\end{array}\right) \in R_{x^{5}}$ and $\left(\begin{array}{cc}a & 0 \\ 0 & d\end{array}\right) \in R_{1}$, we have

$$
\left(\begin{array}{cc}
0 & b \\
0 & 0
\end{array}\right)\left(\begin{array}{cc}
a & 0 \\
0 & d
\end{array}\right)=\left(\begin{array}{cc}
0 & b d \\
0 & 0
\end{array}\right) \in R_{x^{5}}=R_{x^{5} * 1}
$$

(viii) $R_{x^{5}} R_{x} \subseteq R_{x^{5} * x}=R_{1}$, as for all $\left(\begin{array}{cc}0 & b \\ 0 & 0\end{array}\right) \in R_{x^{5}}$ and $\left(\begin{array}{cc}0 & 0 \\ c & 0\end{array}\right) \in R_{x}$, we have

$$
\left(\begin{array}{ll}
0 & b \\
0 & 0
\end{array}\right)\left(\begin{array}{cc}
0 & 0 \\
c & 0
\end{array}\right)=\left(\begin{array}{cc}
b c & 0 \\
0 & 0
\end{array}\right) \in R_{1}=R_{x^{5} * x}
$$

(ix) $R_{x^{5}} R_{x^{5}} \subseteq R_{x^{5} * x^{5}}=R_{x}$, as for all $\left(\begin{array}{cc}0 & b_{1} \\ 0 & 0\end{array}\right),\left(\begin{array}{cc}0 & b_{2} \\ 0 & 0\end{array}\right) \in R_{x^{5}}$, we have

$$
\left(\begin{array}{cc}
0 & b_{1} \\
0 & 0
\end{array}\right)\left(\begin{array}{cc}
0 & b_{2} \\
0 & 0
\end{array}\right)=\left(\begin{array}{cc}
0 & 0 \\
0 & 0
\end{array}\right) \in R_{x}=R_{x^{5} * x^{5}}
$$

Therefore, $R$ is a $\mathfrak{G}$-weak graded ring. However, it is not a fully $\mathfrak{G}$-weak graded ring. For instance, $R_{x^{5}} R_{x^{5}} \neq R_{x^{5} * x^{5}}$ since $R_{x}=R_{x^{5} * x^{5}} \nsubseteq R_{x^{5}} R_{x^{5}}$.

Example 2. Let $X=\left(\mathbb{Z}_{6},+\right)$ and $H=<3>=\{0,3\}$. Choose the set of left coset representatives to be $\mathfrak{G}=\{1,3,5\}$. Then the * and $f$ operations as well as the actions $\triangleleft, \triangleright$ are given by the following tables:

If we consider the Morita ring $T=\left(\begin{array}{cc}R & M \\ N & S\end{array}\right)$, then we have:

$$
T=T_{1} \oplus T_{3} \oplus T_{5},
$$

where

$$
T_{1}=\left(\begin{array}{cc}
0 & M \\
0 & 0
\end{array}\right), T_{3}=\left(\begin{array}{cc}
R & 0 \\
0 & S
\end{array}\right) \text { and } T_{5}=\left(\begin{array}{cc}
0 & 0 \\
N & 0
\end{array}\right)
$$


Table 3: $*$ and $f$ operations.

\begin{tabular}{|l||l|l|l|}
\hline$*$ & 3 & 1 & 5 \\
\hline \hline 3 & 3 & 1 & 5 \\
1 & 1 & 5 & 3 \\
5 & 5 & 3 & 1 \\
\hline
\end{tabular}

\begin{tabular}{|l||l|l|l|}
\hline$f$ & 3 & 1 & 5 \\
\hline \hline 3 & 3 & 3 & 3 \\
1 & 3 & 3 & 3 \\
5 & 3 & 3 & 3 \\
\hline
\end{tabular}

Table 4: $\triangleright$ and $\triangleleft$ actions.

\begin{tabular}{|l||l|l|}
\hline$s \triangleright u$ & 0 & 3 \\
\hline \hline 3 & 0 & 3 \\
1 & 0 & 3 \\
5 & 0 & 3 \\
\hline
\end{tabular}

\begin{tabular}{|l||l|l|}
\hline$s \triangleleft u$ & 0 & 3 \\
\hline \hline 3 & 3 & 3 \\
1 & 1 & 1 \\
5 & 5 & 5 \\
\hline
\end{tabular}

Moreover, the inclusion property $T_{s} T_{t} \subseteq T_{s * t}$ is satisfied for all $s, t \in \mathfrak{G}$ which can illustrated as follows:

(i) $T_{3} T_{3} \subseteq T_{3 * 3}=T_{3}$, as for all $\left(\begin{array}{cc}r_{1} & 0 \\ 0 & s_{1}\end{array}\right)$ and $\left(\begin{array}{cc}r_{2} & 0 \\ 0 & s_{2}\end{array}\right) \in T_{3}$, we have

$$
\left(\begin{array}{cc}
r_{1} & 0 \\
0 & s_{1}
\end{array}\right)\left(\begin{array}{cc}
r_{2} & 0 \\
0 & s_{2}
\end{array}\right)=\left(\begin{array}{cc}
r_{1} r_{2} & 0 \\
0 & s_{1} s_{2}
\end{array}\right) \in T_{3}=T_{3 * 3}
$$

(ii) $T_{3} T_{1} \subseteq T_{3 * 1}=T_{1}$, as for all $\left(\begin{array}{cc}r & 0 \\ 0 & s\end{array}\right) \in T_{3}$ and $\left(\begin{array}{cc}0 & m \\ 0 & 0\end{array}\right) \in T_{1}$, we have

$$
\left(\begin{array}{cc}
r & 0 \\
0 & s
\end{array}\right)\left(\begin{array}{cc}
0 & m \\
0 & 0
\end{array}\right)=\left(\begin{array}{cc}
0 & r m \\
0 & 0
\end{array}\right) \in T_{1}=T_{3 * 1} \text {. }
$$

(iii) $T_{3} T_{5} \subseteq T_{3 * 5}=T_{5}$, as for all $\left(\begin{array}{cc}r & 0 \\ 0 & s\end{array}\right) \in T_{3}$ and $\left(\begin{array}{cc}0 & 0 \\ s & 0\end{array}\right) \in T_{5}$, we have

$$
\left(\begin{array}{cc}
r & 0 \\
0 & s
\end{array}\right)\left(\begin{array}{cc}
0 & 0 \\
s & 0
\end{array}\right)=\left(\begin{array}{cc}
0 & 0 \\
s n & 0
\end{array}\right) \in T_{5}=T_{3 * 5}
$$

(iv) $T_{1} T_{3} \subseteq T_{1 * 3}=T_{1}$, as for all $\left(\begin{array}{cc}0 & m \\ 0 & 0\end{array}\right) \in T_{1}$ and $\left(\begin{array}{cc}r & 0 \\ 0 & s\end{array}\right) \in T_{3}$, we have

$$
\left(\begin{array}{cc}
0 & m \\
0 & 0
\end{array}\right)\left(\begin{array}{cc}
r & 0 \\
0 & s
\end{array}\right)=\left(\begin{array}{cc}
0 & m s \\
0 & 0
\end{array}\right) \in T_{1}=T_{1 * 3} \text {. }
$$

(v) $T_{1} T_{1} \subseteq T_{1 * 1}=T_{5}$, as for all $\left(\begin{array}{cc}0 & m_{1} \\ 0 & 0\end{array}\right)$ and $\left(\begin{array}{cc}0 & m_{2} \\ 0 & 0\end{array}\right) \in T_{1}$, we have

$$
\left(\begin{array}{cc}
0 & m_{1} \\
0 & 0
\end{array}\right)\left(\begin{array}{cc}
0 & m_{2} \\
0 & 0
\end{array}\right)=\left(\begin{array}{cc}
0 & 0 \\
0 & 0
\end{array}\right) \in T_{5}=T_{1 * 1}
$$


(vi) $T_{1} T_{5} \subseteq T_{1 * 5}=T_{3}$, as for all $\left(\begin{array}{cc}0 & m \\ 0 & 0\end{array}\right) \in T_{1}$ and $\left(\begin{array}{cc}0 & 0 \\ n & 0\end{array}\right) \in T_{5}$, we have

$$
\left(\begin{array}{cc}
0 & m \\
0 & 0
\end{array}\right)\left(\begin{array}{ll}
0 & 0 \\
n & 0
\end{array}\right)=\left(\begin{array}{cc}
m n & 0 \\
0 & 0
\end{array}\right) \in T_{3}=T_{1 * 5}
$$

(vii) $T_{5} T_{3} \subseteq T_{5 * 3}=T_{5}$, as for all $\left(\begin{array}{ll}0 & 0 \\ n & 0\end{array}\right) \in T_{5}$ and $\left(\begin{array}{cc}r & 0 \\ 0 & s\end{array}\right) \in T_{3}$, we have

$$
\left(\begin{array}{ll}
0 & 0 \\
n & 0
\end{array}\right)\left(\begin{array}{ll}
r & 0 \\
0 & s
\end{array}\right)=\left(\begin{array}{cc}
0 & 0 \\
n r & 0
\end{array}\right) \in T_{5}=T_{5 * 3}
$$

(viii) $T_{5} T_{1} \subseteq T_{5 * 1}=T_{3}$, as for all $\left(\begin{array}{cc}0 & 0 \\ n & 0\end{array}\right) \in T_{5}$ and $\left(\begin{array}{cc}0 & m \\ 0 & 0\end{array}\right) \in T_{1}$, we have

$$
\left(\begin{array}{cc}
0 & 0 \\
n & 0
\end{array}\right)\left(\begin{array}{cc}
0 & m \\
0 & 0
\end{array}\right)=\left(\begin{array}{cc}
0 & 0 \\
0 & m n
\end{array}\right) \in T_{3}=T_{5 * 1} \text {. }
$$

(ix) $T_{5} T_{5} \subseteq T_{5 * 5}=T_{1}$, as for all $\left(\begin{array}{cc}0 & 0 \\ n_{1} & 0\end{array}\right)$ and $\left(\begin{array}{cc}0 & 0 \\ n_{2} & 0\end{array}\right) \in T_{5}$, we have

$$
\left(\begin{array}{cc}
0 & 0 \\
n_{1} & 0
\end{array}\right)\left(\begin{array}{cc}
0 & 0 \\
n_{2} & 0
\end{array}\right)=\left(\begin{array}{cc}
0 & 0 \\
0 & 0
\end{array}\right) \in T_{1}=T_{5 * 5}
$$

Thus, $T$ is a $\mathfrak{G}$-weak graded ring. However, it is not a fully $\mathfrak{G}$-weak graded ring. For instance, $T_{5} T_{5} \neq T_{5 * 5}$ since $T_{1}=T_{5 * 5} \nsubseteq T_{5} T_{5}$.

Example 3. Consider the ring of real quaternions $(\mathbb{H},+, \cdot)$. Let

$$
X=D_{6}=\left\{1, x, x^{2}, x^{3}, x^{4}, x^{5}, y, x y, x^{2} y, x^{3} y, x^{4} y, x^{5} y\right\}
$$

and $H=\left\{1, x^{2}, x^{4}\right\}$ be an additive subgroup of the group $X$. Take the set of left coset representatives to be $\mathfrak{G}=\left\{1, y, x^{5}, x y\right\}$. Then the $*$ and $f$ operations as well as the actions $\triangleleft, \triangleright$ are given by the following tables:

Table 5: $*$ and $f$ operations.

\begin{tabular}{|l||l|l|l|l|}
\hline$*$ & 1 & $y$ & $x^{5}$ & $x y$ \\
\hline \hline 1 & 1 & $y$ & $x^{5}$ & $x y$ \\
$y$ & $y$ & 1 & $x y$ & $x^{5}$ \\
$x^{5}$ & $x^{5}$ & $x y$ & 1 & $y$ \\
$x y$ & $x y$ & $x^{5}$ & $y$ & 1 \\
\hline
\end{tabular}

\begin{tabular}{|l||l|l|l|l|}
\hline$f$ & 1 & $y$ & $x^{5}$ & $x y$ \\
\hline \hline 1 & 1 & 1 & 1 & 1 \\
$y$ & 1 & 1 & 1 & 1 \\
$x^{5}$ & 1 & $x^{4}$ & $x^{4}$ & 1 \\
$x y$ & 1 & $x^{2}$ & $x^{2}$ & 1 \\
\hline
\end{tabular}


Table 6: $\triangleright$ and $\triangleleft$ actions.

\begin{tabular}{|l||l|l|l|}
\hline$s \triangleright u$ & 1 & $x^{2}$ & $x^{4}$ \\
\hline \hline 1 & 1 & $x^{2}$ & $x^{4}$ \\
$y$ & 1 & $x^{4}$ & $x^{2}$ \\
$x^{5}$ & 1 & $x^{2}$ & $x^{4}$ \\
$x y$ & 1 & $x^{4}$ & $x^{2}$ \\
\hline
\end{tabular}

\begin{tabular}{|l||l|l|l|}
\hline$s \triangleleft u$ & 1 & $x^{2}$ & $x^{4}$ \\
\hline \hline 1 & 1 & 1 & 1 \\
$y$ & $y$ & $y$ & $y$ \\
$x^{5}$ & $x^{5}$ & $x^{5}$ & $x^{5}$ \\
$x y$ & $x y$ & $x y$ & $x y$ \\
\hline
\end{tabular}

Thus, $\mathbb{H}=R_{1} \oplus R_{y} \oplus R_{x^{5}} \oplus R_{x y}$ where, $R_{1}=\mathbb{R}, R_{y}=\mathbb{R} i, R_{x^{5}}=\mathbb{R} j$ and $R_{x y}=\mathbb{R} k$.

The inclusion property can be checked as follows for any $r, r^{\prime} \in \mathbb{R}$ :

(i) $R_{1} R_{1} \subseteq R_{1 * 1}=R_{1}$, as for all $r, r^{\prime} \in R_{1}$, we have $(r)\left(r^{\prime}\right)=r r^{\prime} \in R_{1}=R_{1 * 1}$.

(ii) $R_{1} R_{y} \subseteq R_{1 * y}=R_{y}$, as for all $r \in R_{1}, r^{\prime} i \in R_{y}$, we have $(r)\left(r^{\prime} i\right)=\left(r r^{\prime}\right) i \in R_{y}=R_{1 * y}$

(iii) $R_{1} R_{x^{5}} \subseteq R_{1 * x^{5}}=R_{x^{5}}$, as for all $r \in R_{1}, r^{\prime} j \in R_{x^{5}}$, we have $(r)\left(r^{\prime} j\right)=\left(r r^{\prime}\right) j \in R_{x^{5}}=R_{1 * x^{5}}$.

(iv) $R_{1} R_{x y} \subseteq R_{1 * x y}=R_{x y}$, as for all $r \in R_{1}, r^{\prime} k \in R_{x y}$, we have $(r)\left(r^{\prime} k\right)=\left(r r^{\prime}\right) k \in R_{x y}=R_{1 * x y}$.

(v) $R_{y} R_{1} \subseteq R_{y * 1}=R_{y}$, as for all $r i \in R_{y}, r^{\prime} \in R_{1}$, we have $(r i)\left(r^{\prime}\right)=\left(r r^{\prime}\right) i \in R_{y}=R_{y * 1}$.

(vi) $R_{y} R_{y} \subseteq R_{y * y}=R_{1}$, as for all $r i, r^{\prime} i \in R_{y}$, we have $(r i)\left(r^{\prime} i\right)=\left(-r r^{\prime}\right) \in R_{1}=R_{y * y}$.

(vii) $R_{y} R_{x^{5}} \subseteq R_{y * x^{5}}=R_{x y}$, as for all $r i \in R_{y}, r^{\prime} j \in R_{x^{5}}$, we have $(r i)\left(r^{\prime} j\right)=\left(r r^{\prime}\right) k \in R_{x y}=R_{y * x^{5}}$.

(viii) $R_{y} R_{x y} \subseteq R_{y * x y}=R_{x^{5}}$, as for all $r i \in R_{y}, r^{\prime} k \in R_{x y}$, we have $(r i)\left(r^{\prime} k\right)=\left(-r r^{\prime}\right) j \in R_{x^{5}}=R_{y * x y}$.

(ix) $R_{x^{5}} R_{1} \subseteq R_{x^{5} * 1}=R_{x^{5}}$, as for all $r j \in R_{x^{5}}, r^{\prime} \in R_{1}$, we have $(r j)\left(r^{\prime}\right)=\left(r r^{\prime}\right) j \in R_{x^{5}}=R_{x^{5} * 1}$.

(x) $R_{x^{5}} R_{y} \subseteq R_{x^{5} * y}=R_{x y}$, as for all $r j \in R_{x^{5}}, r^{\prime} i \in R_{y}$, we have $(r j)\left(r^{\prime} i\right)=\left(-r r^{\prime}\right) k \in R_{x y}=R_{x^{5} * y}$.

(xi) $R_{x^{5}} R_{x^{5}} \subseteq R_{x^{5} * x^{5}}=R_{1}$, as for all $r j, r^{\prime} j \in R_{x^{5}}$, we have $(r j)\left(r^{\prime} j\right)=\left(-r r^{\prime}\right) \in R_{1}=R_{x^{5} * x^{5}}$.

(xii) $R_{x^{5}} R_{x y} \subseteq R_{x^{5} * x y}=R_{y}$, as for all $r j \in R_{x^{5}}, r^{\prime} k \in R_{x y}$, we have $(r j)\left(r^{\prime} k\right)=\left(r r^{\prime}\right) i \in R_{y}=R_{x^{5} * x y}$

(xiii) $R_{x y} R_{1} \subseteq R_{x y * 1}=R_{x y}$, as for all $r k \in R_{x y}, r^{\prime} \in R_{1}$, we have $(r k)\left(r^{\prime}\right)=\left(r r^{\prime}\right) k \in R_{x y}=R_{x y * 1}$. 
(xiv) $R_{x y} R_{y} \subseteq R_{x y * y}=R_{x^{5}}$, as for all $r k \in R_{x y}, r^{\prime} i \in R_{y}$, we have $(r k)\left(r^{\prime} i\right)=\left(r r^{\prime}\right) j \in R_{x^{5}}=R_{x y * y}$.

(xv) $R_{x y} R_{x^{5}} \subseteq R_{x y * x^{5}}=R_{y}$, as for all $r k \in R_{x y}, r^{\prime} j \in R_{x^{5}}$, we have $(r k)\left(r^{\prime} j\right)=\left(-r r^{\prime}\right) i \in R_{y}=R_{x y * x^{5}}$.

(xvi) $R_{x y} R_{x y} \subseteq R_{x y * x y}=R_{1}$, as for all $r k, r^{\prime} k \in R_{x y}$, we have $(r k)\left(r^{\prime} k\right)=\left(-r r^{\prime}\right) \in R_{1}=R_{x y * x y}$.

Therefore, $\mathbb{H}$ is a fully $\mathfrak{G}$-weak graded ring.

\section{References}

[1] G. Abrams and C. Menini. Rings of endomorphisms of semigroup-graded modules. Rocky Mountain J. Math., 26(2):375-406, (1996).

[2] T. Albu, C. Năstăsescu, Infinite group-graded rings, rings of endomorphisms and localization. J. Pure Appl. Algebra, 59:125-150, (1989).

[3] M. M. Al-Shomrani. A construction of graded rings using a set of left coset representatives. JP Journal of Algebra, Number Theory and Applications, 25(2):133-144, (2012).

[4] N. Al-Subaie, M. Al-Shomrani, On weak graded rings. Eur. J. Pure Appl. Math., 10:967-980, (2017).

[5] M. Beattie, Duality theorems for rings with actions and coactions. J. Algebra, 115:302-312, (1988).

[6] E. J. Beggs. Making non-trivially associated tensor categories from left coset representatives. J. Pure Appl. Algebra, 177(1):5-41, (2003).

[7] M. Cohen and S. Montgomery. Group-graded rings, smash product and group actions. Trans. Amer. Math. Soc., 282(1):237-258, (1984).

[8] E. C. Dade. Group graded rings and modules. Math. Z., 174:241-262, (1980).

[9] E. Dade, Clifford theory for group graded rings. J. Reine Angew. Math., 369:40-86, (1986).

[10] E. Dade, Clifford theory for group graded rings II. J. Reine Angew. Math., 387:148181, (1988).

[11] S. Dascalescu, A. V. Kelarev and L. Van Wyk. Semigroup gradings of full matrix rings. Comm. Algebra, 29(11):5023-5031, (2001).

[12] J. L. Gómez Pardo and C. Năstăsescu. Relative projectivity, graded Cliffored theory and applications. J. Algebra, 141(2):484-504, (1991). 
[13] G. Karpilovsky. The Jacobson radical of monoid-graded algebras. Tsukuba J. Math, 16(1):19-52, (1992).

[14] A. V. Kelarev. Applications of epigroups to graded ring theory. Semigroup Forum, 50(3):327-350, (1995).

[15] A. V. Kelarev. Semisimple rings graded by inverse semigroups. J. Algebra, 205:451459, (1998).

[16] C. Năstăsescu, F. Oystaeyen, Graded Ring Theory. North-Holland Publishing Company, (1982).

[17] C. Năstăsescu, M. Bergh, F. Oystaeyen, Separable functor, applications to graded rings and modules. J. Algebra, 123:397-413, (1989).

[18] C. Năstăsescu and F. V. Oystaeyen. Methods of Graded Rings. Springer-Verlag Berlin Heidelberg, New York, (2004).

[19] P. Nystedt and J. Oinert. Simple semigroup graded rings. J. Algebra Appl., 14(7):110, (2015).

[20] M. Rafael, J. Oinert, Separable functors revisited. Comm. Algebra, 18:1441459,(1990).

[21] A. Turull, Clifford theory with Schur Indices. J. Algebra, 170:661-677, (1994).

[22] B. Zhou, Two Clifford's theorems for strongly group-graded rings. J. Algebra, 139:172-189, (1991). 